\title{
Recent perturbative results on heavy quark decays
}

\author{
Jan Piclum
}

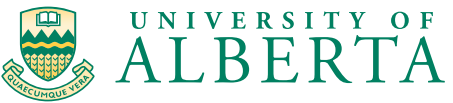




\section{Outline}

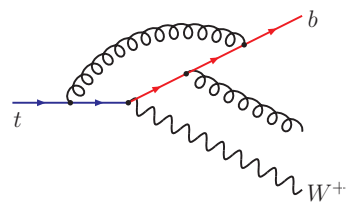

(3) Helicity fractions of $W$ bosons from top quark decays JHP, Andrzej Czarnecki, Jürgen Körner

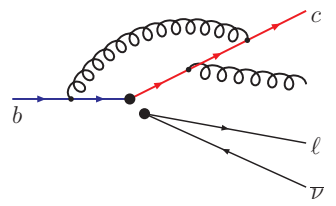

(2) Mass effects in semi-leptonic bottom quark decays Alexey Pak, Andrzej Czarnecki 
Part I

\section{Helicity fractions}

JHP, Andrzej Czarnecki, Jürgen Körner 


\section{Introduction}

- couplings are very susceptible to "new physics"

- dominant decay mode: $t \rightarrow b+W^{+}$

- polarisation allows for detailed study of decay process

- LHC will produce many top quarks

- total width is hard to measure directly at hadron colliders

- helicity fractions can be measured 
00000

\section{$t \rightarrow b+W^{+}$}

$W$ has spin $1 \rightsquigarrow 3$ polarisation states

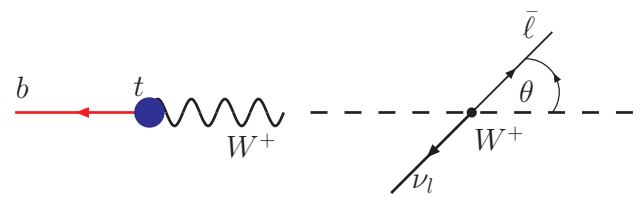

$\rightsquigarrow$ different contributions to angular distribution

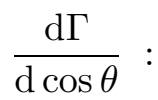

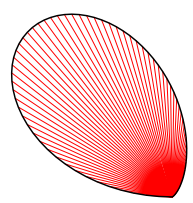

W

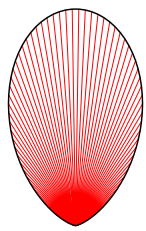

$\mathrm{W}_{L}$

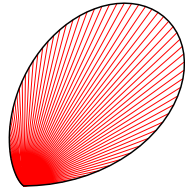

$\mathrm{W}_{+}$ 


\section{Effect of radiative corrections}

bottom quark has to be left-handed
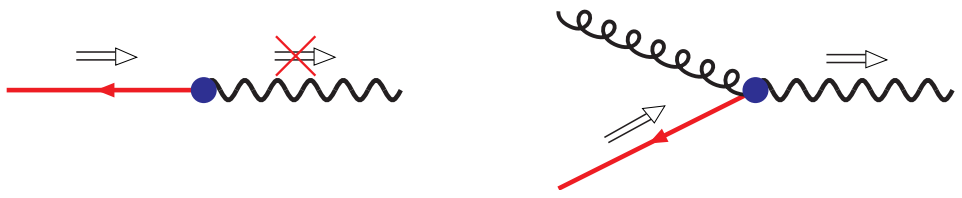

additional gluon and $m_{b} \neq 0$ leads to $\Gamma_{+} \neq 0$ 


\section{Experimental results}

leading order Standard Model predictions $\left(m_{b}=0\right)$

$$
\mathcal{F}_{L}=0.697, \mathcal{F}_{-}=0.303, \mathcal{F}_{+}=0
$$

Tevatron

[CDF '07] [D0 '05, '07]

$$
\begin{aligned}
& \mathcal{F}_{L}=0.85 \pm 0.28, \mathcal{F}_{+}=0.05 \pm 0.12 \\
& \mathcal{F}_{L}=0.56 \pm 0.31, \mathcal{F}_{+}=0.056 \pm 0.10
\end{aligned}
$$

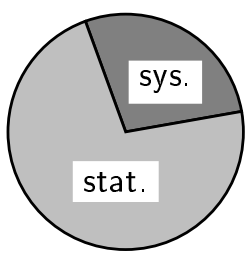

LHC with $10 \mathrm{fb}^{-1}$

[Aguilar-Saavedra et al. '07]

$$
\Delta \mathcal{F}_{L}=1.9 \%, \Delta \mathcal{F}_{-}=1.8 \%, \Delta \mathcal{F}_{+}=0.21 \%
$$




\section{State of the art}

\section{unpolarised decay}

$\mathcal{O}\left(\alpha_{s}\right)$ correction with full $m_{b}$ and $m_{W}$ dependence $\mathcal{O}\left(\alpha_{s}^{2}\right)$ correction as expansion in $m_{W} / m_{t}$
Jeżabek

Kühn

Czarnecki

Blokland

Ślusarczyk

Tkachov

Pak

\section{polarised decay}

$\mathcal{O}\left(\alpha_{s}\right)$ correction with full $m_{b}$ and $m_{W}$ dependence subsequent decay of $\mathrm{W}$ boson 


\section{How can we reach $\mathcal{O}\left(\alpha_{s}^{2}\right)$ for polarised decay?}

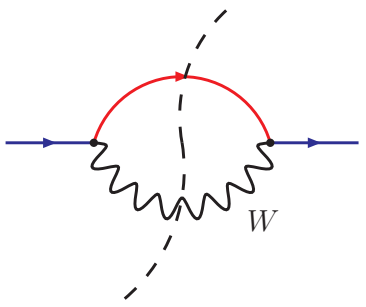

apply optical theorem and expansion by regions do not sum over polarisations 


\section{How can we reach $\mathcal{O}\left(\alpha_{s}^{2}\right)$ for polarised decay?}

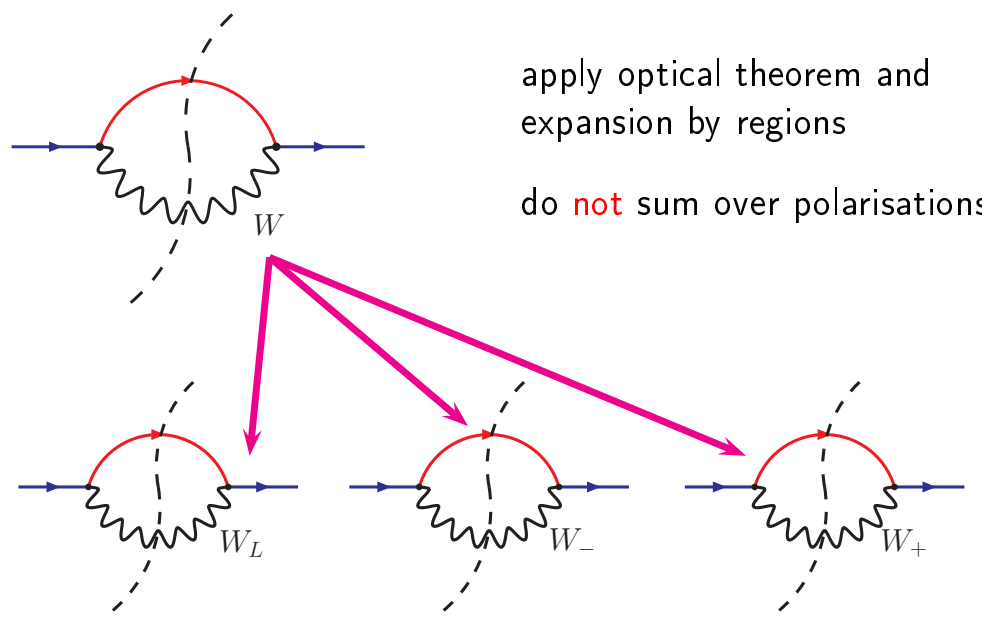




\section{3-loop diagrams}
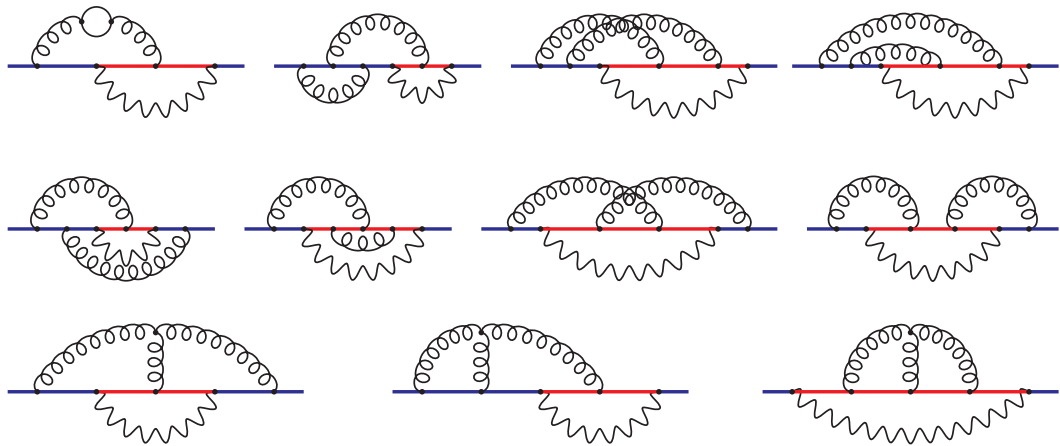

$m_{b}=0 \rightsquigarrow$ two scales: $m_{t}, m_{W}$

$\rightsquigarrow$ use expansion by regions to reduce to single scale integrals 
Introduction

Calculation

Results

00000

Expansion by regions

on-shell integrals with two masses and large mass on-shell $\rightsquigarrow$ two regions:

- hard: $q_{i} \sim m_{t} \rightsquigarrow$ expand in $m_{W}$

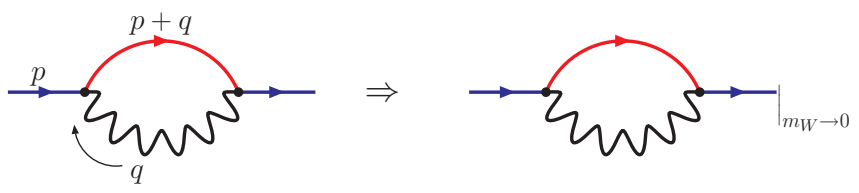

- soft: $q_{i} \sim m_{W} \rightsquigarrow$ expand in $q$

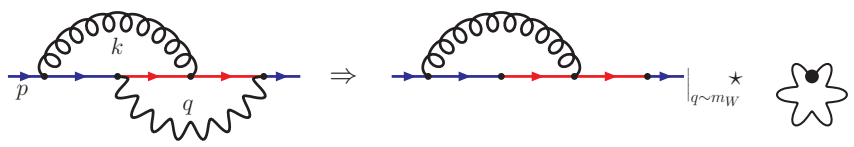




\section{How does polarisation enter the game?}

\section{polarisation sum}

$$
\epsilon_{L \mu}^{*} \epsilon_{L \nu}+\epsilon_{+\mu}^{*} \epsilon_{+\nu}+\epsilon_{-\mu}^{*} \epsilon_{-\nu}=-g_{\mu \nu}+\frac{q_{\mu} q_{\nu}}{m_{W}^{2}}
$$

$\rightsquigarrow$ numerator of $\mathrm{W}$ boson propagator

pick out individual terms:

$$
\epsilon_{L \mu}^{*} \epsilon_{L \nu}=\frac{\left(m_{W}^{2} p^{\mu}-p \cdot q q^{\mu}\right)\left(m_{W}^{2} p^{\nu}-p \cdot q q^{\nu}\right)}{m_{W}^{2} m_{t}^{2}|\vec{q}|^{2}}
$$




\section{How does polarisation enter the game?}

\section{polarisation sum}

$$
\epsilon_{L \mu}^{*} \epsilon_{L \nu}+\epsilon_{+\mu}^{*} \epsilon_{+\nu}+\epsilon_{-\mu}^{*} \epsilon_{-\nu}=-g_{\mu \nu}+\frac{q_{\mu} q_{\nu}}{m_{W}^{2}}
$$

$\rightsquigarrow$ numerator of $\mathrm{W}$ boson propagator

pick out individual terms:

$$
\epsilon_{L \mu}^{*} \epsilon_{L \nu}=\frac{\left(m_{W}^{2} p^{\mu}-p \cdot q q^{\mu}\right)\left({ }^{2}{ }_{W} p^{\nu}-p \cdot q q^{\nu}\right)}{m_{W}^{2} \eta\left({ }_{t}^{2}|\vec{q}|^{2}\right)}
$$

complication: appearance of $|\vec{q}|=\sqrt{q_{0}^{2}-m_{W}^{2}}$ in denominator 


\section{Soft region}

$\rightsquigarrow|\vec{q}|^{2}=q_{0}^{2}-m_{W}^{2}$ cannot be expanded, but W loop factorises:
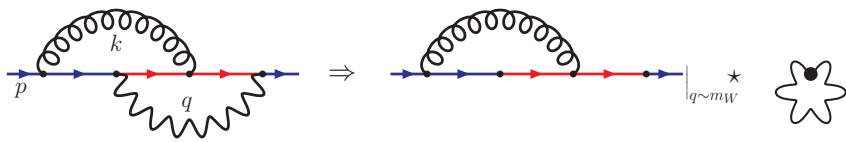

$\rightsquigarrow$ calculation just as in unpolarised case, but with different vacuum integral:

$$
\int \frac{\mathrm{d}^{d} q}{\left(q^{2}-m_{W}^{2}\right)\left(q_{0}^{2}-m_{W}^{2}\right)}=i \pi^{d / 2} \frac{2 \Gamma(\epsilon)}{1-2 \epsilon} \frac{1}{\left(m_{W}^{2}\right)^{\epsilon}}
$$




\section{Hard region}

rewrite $|\vec{q}|^{2}$ in terms of $p^{\mu}$ and $q^{\mu}$

$$
|\vec{q}|^{2}=q_{0}^{2}-m_{W}^{2}=\frac{(2 p \cdot q)^{2}}{4 m_{t}^{2}}-m_{W}^{2}
$$

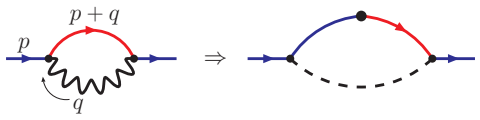

we only need the imaginary part $\rightsquigarrow q^{2}=m_{W}^{2}$ :

$$
|\vec{q}|^{2}=\frac{1}{4 m_{t}^{2}}\left(q^{2}+2 p \cdot q-m_{W}^{2}\right)^{2}-m_{W}^{2}
$$

\section{expansion in terms of top propagators}

$$
\frac{1}{|\vec{q}|^{2}}=\frac{4 m_{t}^{2}}{\left(q^{2}+2 p \cdot q\right)^{2}} \sum_{i=0}^{\infty}\left(\frac{\ldots}{\left(q^{2}+2 p \cdot q\right)^{2}}\right)^{i}
$$




\section{Results}

- checked expansion of $\mathcal{O}\left(\alpha_{s}\right)$ up to $\left(m_{W} / m_{t}\right)^{16}$

- obtained analytical results for all three-loop master integrals

- calculated $\mathcal{O}\left(\alpha_{s}^{2}\right)$ corrections up to $\left(m_{W} / m_{t}\right)^{10}$ 


\section{Numerical evaluation}

\section{Helicity fractions}

$$
\begin{aligned}
& \mathcal{F}_{L}=0.6971(1-0.0108-0.0034)=0.6872(6) \\
& \mathcal{F}_{-}=0.3029(1+0.0214-0.0070)=0.3115(5) \\
& \mathcal{F}_{+}=0+0.00103+0.00023=0.00126(6)
\end{aligned}
$$

estimated error from expansion: $\sim 10^{-6}$

expected uncertainties at LHC:

$$
\Delta \mathcal{F}_{L}=1.9 \%, \Delta \mathcal{F}_{-}=1.8 \%, \Delta \mathcal{F}_{+}=0.21 \%
$$




\section{$\mathcal{O}\left(\alpha_{s}^{2}\right)$ correction as function of $m_{W} / m_{t}$}

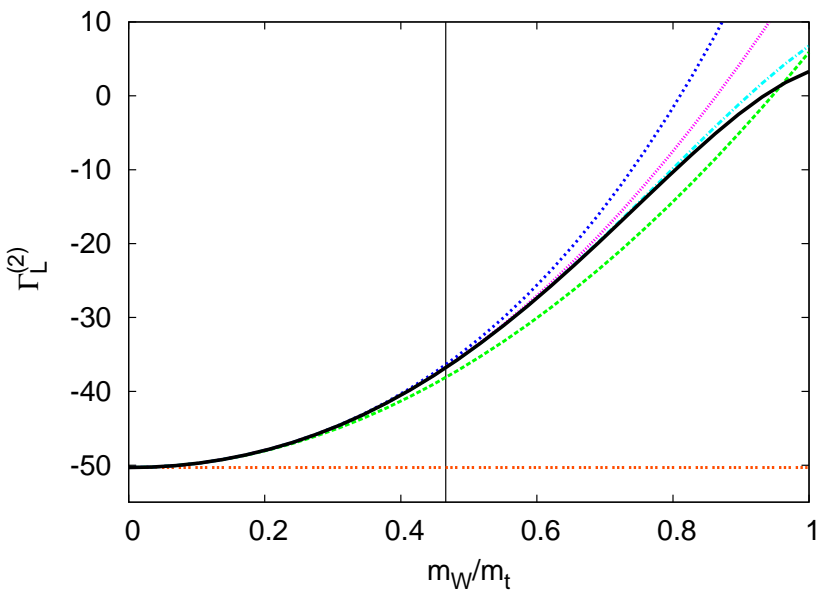

$\frac{\Gamma_{L}^{(2)}(0.466)}{\alpha_{s}^{2} C_{F} G_{0}}=-50.316+12.214+1.736-0.381-0.044-0.003+\ldots$ 


\section{$\mathcal{O}\left(\alpha_{s}^{2}\right)$ correction as function of $m_{W} / m_{t}$}

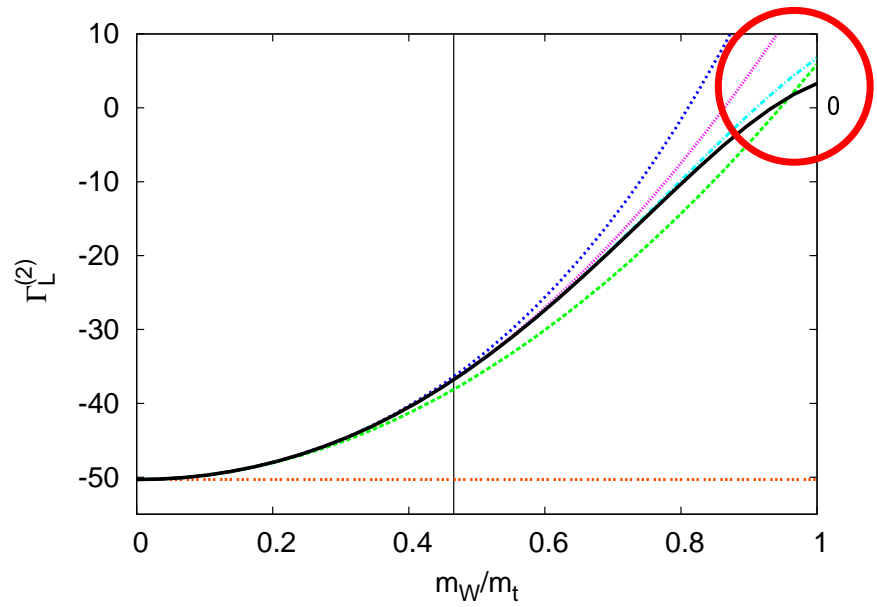

$\frac{\Gamma_{L}^{(2)}(0.466)}{\alpha_{s}^{2} C_{F} G_{0}}=-50.316+12.214+1.736-0.381-0.044-0.003+\ldots$ 


\title{
Part II
}

\section{Mass effects in semi-leptonic $b$ decays}

\author{
Alexey Pak, Andrzej Czarnecki \\ arXiv: 0803.0960 [hep-ph]
}




\section{Introduction}

\section{Semi-leptonic B-meson decay}

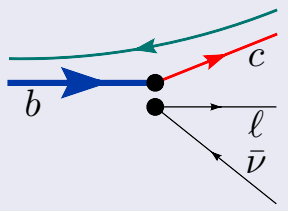

- Clean decay

- HQET: $\Gamma(b \rightarrow c \ell \bar{\nu})$

- $\mathcal{O}\left(\alpha_{s}^{2}\right) \Rightarrow\left|\mathbf{V}_{\mathbf{c b}}\right|, \ldots$

- $m_{c} / M_{b} \sim 0.3$ 


\section{Introduction}

\section{Semi-leptonic B-meson decay}

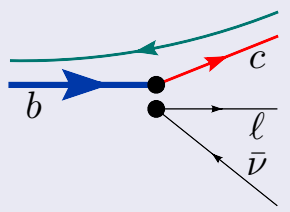

- Clean decay

- HQET: $\Gamma(b \rightarrow c \ell \bar{\nu})$

- $\mathcal{O}\left(\alpha_{s}^{2}\right) \Rightarrow\left|\mathbf{V}_{\text {cb }}\right|, \ldots$

- $m_{c} / M_{b} \sim 0.3$

\section{Muon decay}

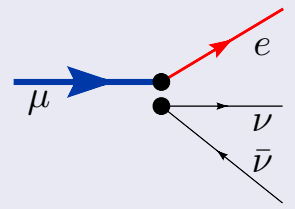

- $\Gamma(\mu \rightarrow e \nu \bar{\nu}) \sim \mathrm{G}_{\mathbf{F}}^{2}$

- MuLan@PSI: <ppm

- Need $\mathcal{O}\left(\alpha^{2}\right)$

- $m_{e} / M_{\mu} \sim 0.005$ 


\section{Studies of mass effects at NNLO}
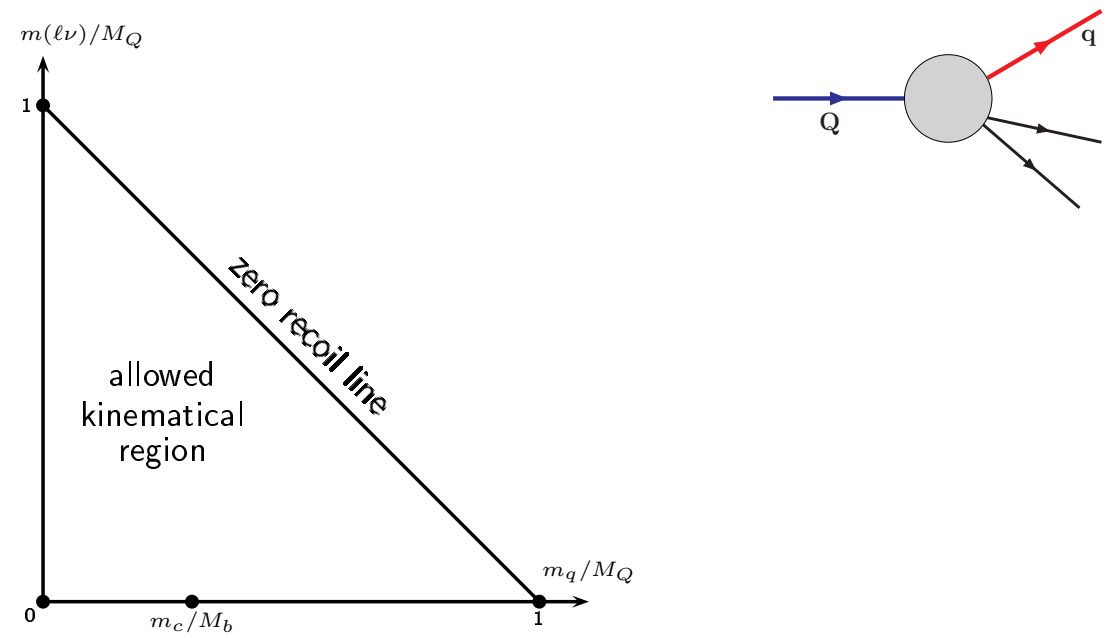


\section{Studies of mass effects at NNLO}
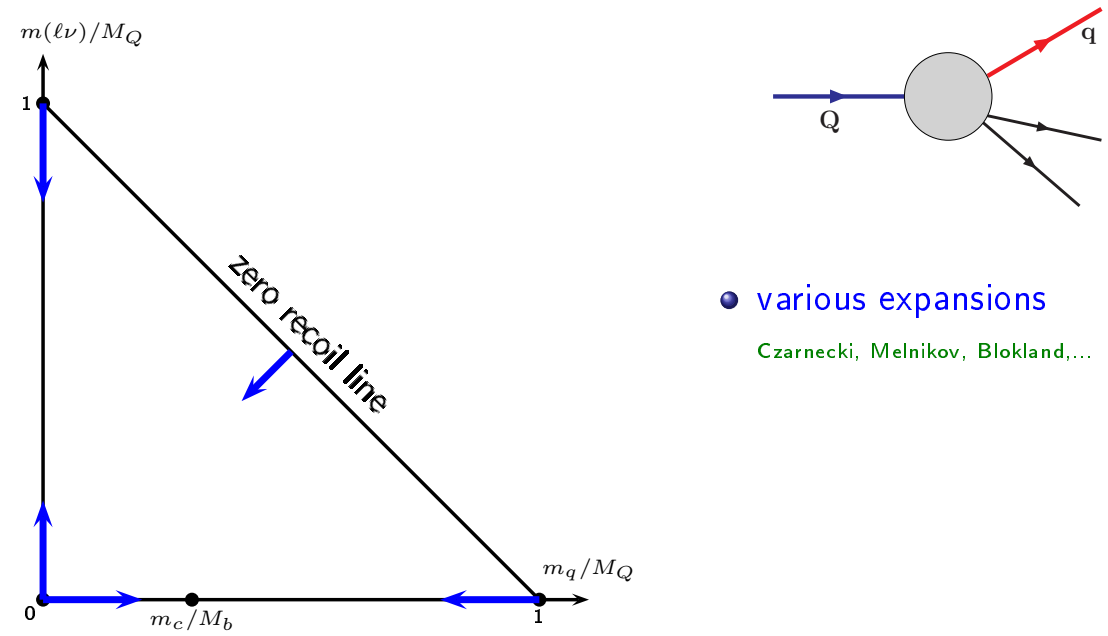

- various expansions

Czarnecki, Melnikov, Blokland,... 


\section{Studies of mass effects at NNLO}
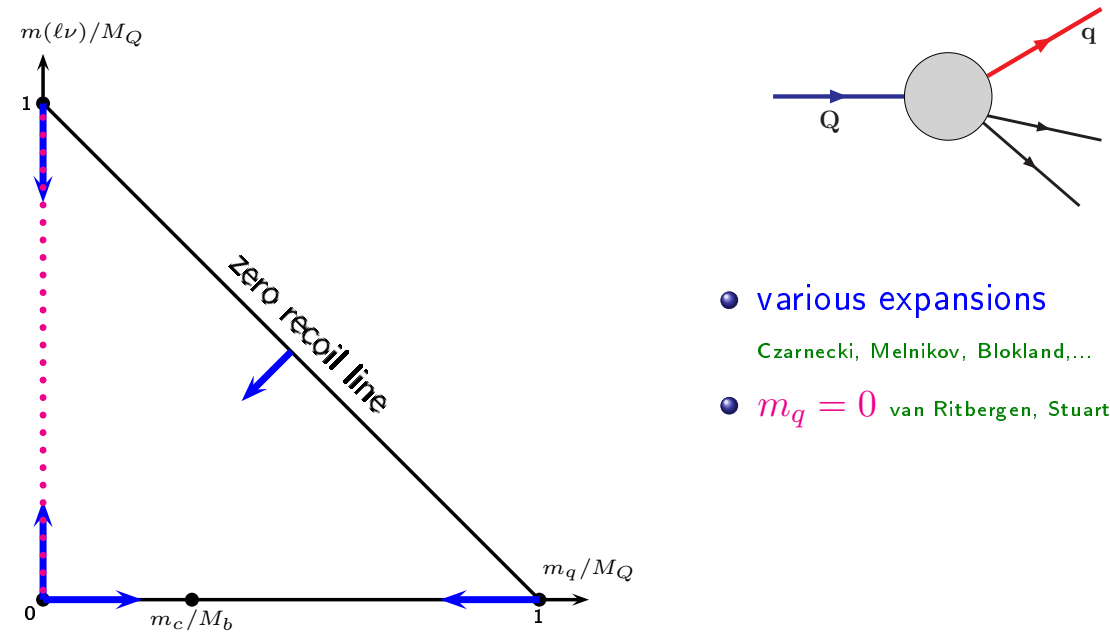

- various expansions

Czarnecki, Melnikov, Blokland,...

- $m_{q}=0$ van Ritbergen, Stuart 


\section{Studies of mass effects at NNLO}
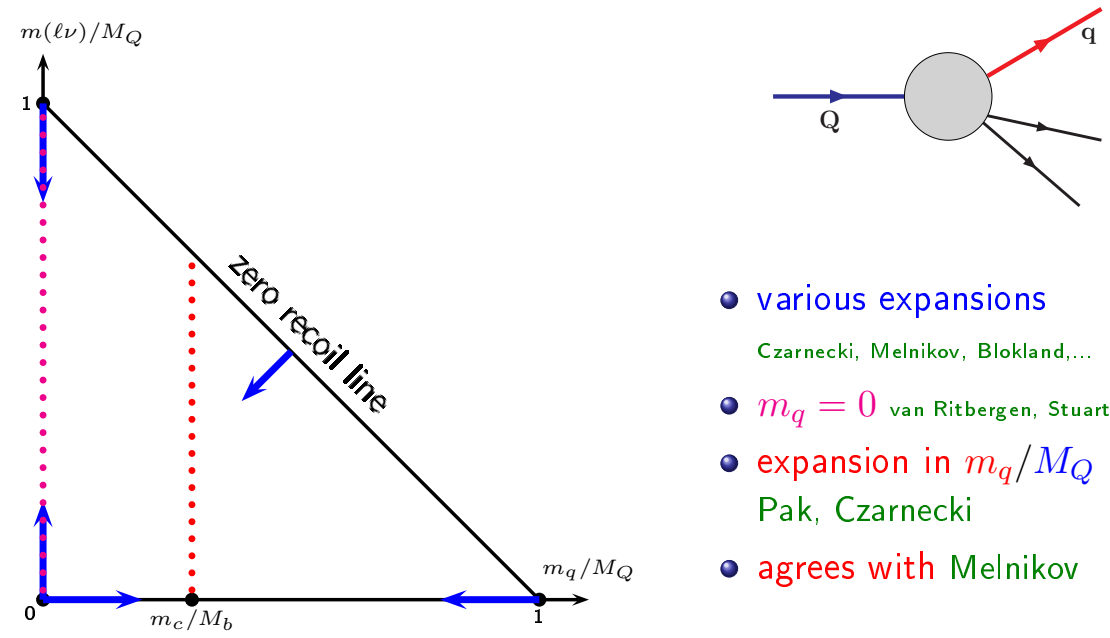

- various expansions

Czarnecki, Melnikov, Blokland,...

- $m_{q}=0$ van Ritbergen, Stuart

- expansion in $m_{q} / M_{Q}$ Pak, Czarnecki

- agrees with Melnikov 


\section{Structure of the result}

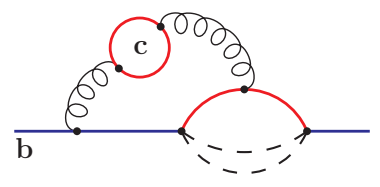

$$
\begin{aligned}
X_{c}= & -\frac{1009}{288}+\frac{8}{3} \zeta_{3}+\frac{77}{216} \pi^{2} \\
& -\rho \quad\left\{\frac{5}{4} \pi^{2}\right\} \\
& +\rho^{2}\left\{\frac{145}{3}+\frac{16}{3} \pi^{2}+\frac{52}{3} \ln \rho-8 \ln ^{2} \rho\right\} \\
& +\rho^{3}\left\{\frac{569}{36} \pi^{2}+\frac{64}{3} \pi^{2} \ln \rho\right\} \\
& +\rho^{4}\left\{-\frac{4483}{36}+196 \zeta_{3}+\frac{\pi^{2}}{6}+\left[\frac{73}{3} \pi^{2}+\frac{599}{6}\right] \ln \rho+44 \ln ^{2} \rho-32 \ln ^{3} \rho\right\} \\
& +\ldots
\end{aligned}
$$




\section{Structure of the result}

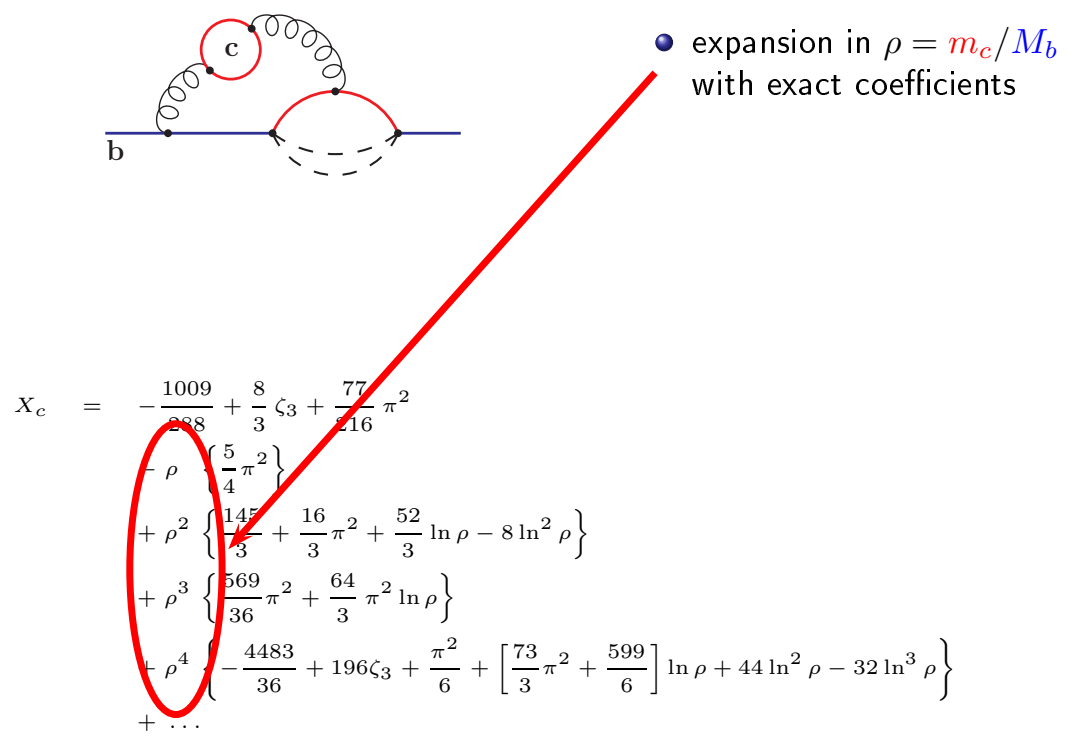




\section{Structure of the result}

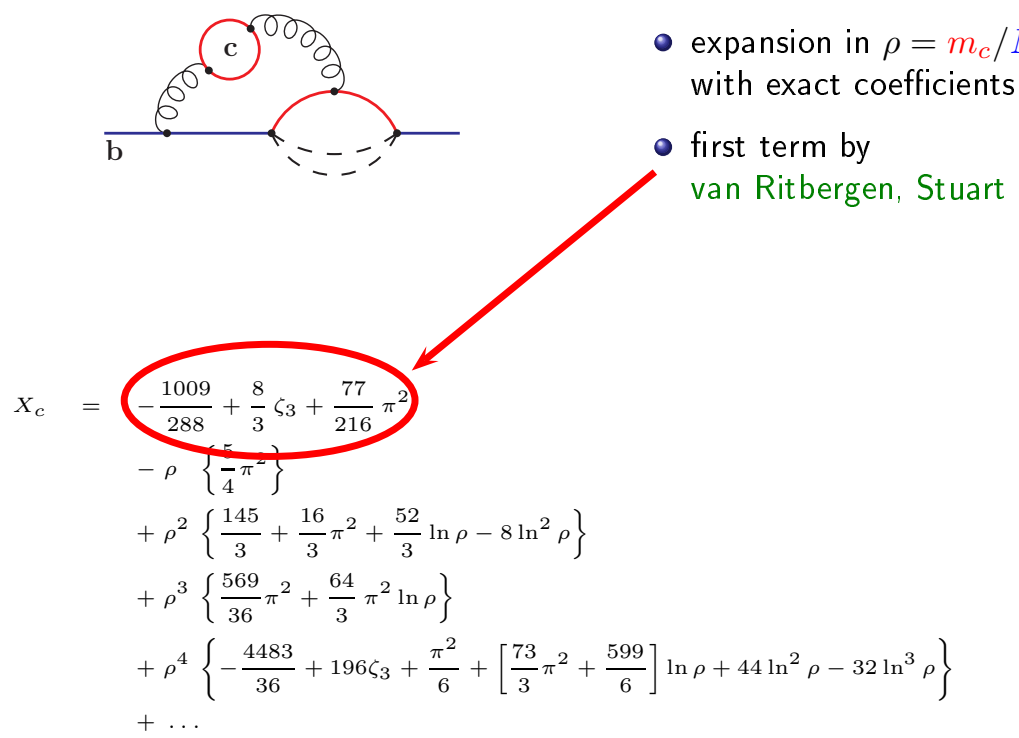




\section{Structure of the result}

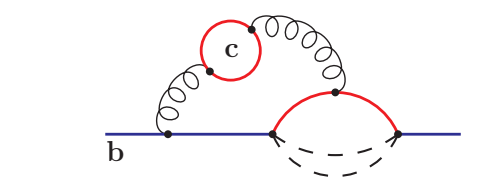

- expansion in $\rho=m_{c} / M_{b}$ with exact coefficients

- first term by van Ritbergen, Stuart

- now calculated through $\rho^{7}$

$X_{c}=-\frac{1009}{288}+\frac{8}{3} \zeta_{3}+\frac{77}{216} \pi^{2}$

$-\rho\left\{\frac{5}{4} \pi^{2}\right\}$

$+\rho^{2}\left\{\frac{145}{3}+\frac{16}{3} \pi^{2}+\frac{52}{3} \ln \rho-8 \ln ^{2} \rho\right\}$

$+\rho^{3}\left\{\frac{569}{36} \pi^{2}+\frac{64}{3} \pi^{2} \ln \rho\right\}$

$+\rho^{4}\left\{-\frac{448}{36}+196 \zeta_{3}+\frac{\pi^{2}}{6}+\left[\frac{73}{3} \pi^{2}+\frac{599}{6}\right] \ln \rho+44 \ln ^{2} \rho-32 \ln ^{3} \rho\right\}$ Pak, Czarnecki 


\section{Structure of the result}

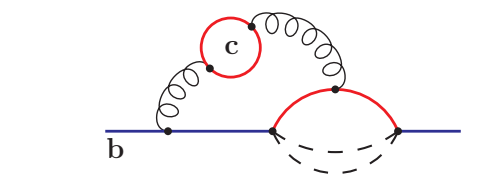

- expansion in $\rho=m_{c} / M_{b}$ with exact coefficients

- first term by van Ritbergen, Stuart

- now calculated through $\rho^{7}$ Pak, Czarnecki

- some logs were known $X_{c}=-\frac{1009}{288}+\frac{8}{3} \zeta_{3}+\frac{77}{216} \pi^{2}$ Bauer, Falk, Luke

$-\rho\left\{\frac{5}{4} \pi^{2}\right\}$

$+\rho^{2}\left\{\frac{145}{3}+\frac{16}{3} \pi^{2}+\frac{52}{3} \ln \right.$

$+\rho^{3}\left\{\frac{569}{36} \pi^{2} \div \frac{64}{3} \pi^{2} \ln \rho\right\}$

$+\rho^{4}\left\{-\frac{4483}{36}+196 \zeta_{3}+\frac{\pi^{2}}{6}+\left[\frac{73}{3} \pi^{2}+\frac{599}{6}\right] \ln \rho+44 \ln ^{2} \rho-32 \ln ^{3} \rho\right\}$ $+\ldots$ 


\section{Structure of the result}

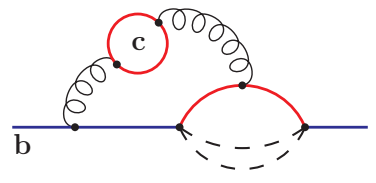

$$
X_{c}=-\frac{1009}{288}+\frac{8}{3} \zeta_{3}+\frac{77}{216} \pi^{2}
$$$$
-\rho\left\{\frac{5}{4} \pi^{2}\right\}
$$$$
+\rho^{2}\left\{\frac{145}{3}+\frac{16}{3} \pi^{2}+\frac{52}{3} \ln \rho-8 \ln ^{2} \rho\right\}
$$$$
+\rho^{3}\left\{\frac{569}{36} \pi^{2}+\frac{64}{3} \pi^{2} \ln \rho\right\}
$$$$
+\rho^{4}\left\{-\frac{4483}{36}+196 \zeta_{3}+\frac{\pi^{2}}{6}+\left[\frac{73}{3} \pi^{2}+\frac{599}{6}\right] \ln \rho+44 \ln ^{2} \rho-32 \ln ^{3} \rho\right.
$$

- expansion in $\rho=m_{c} / M_{b}$ with exact coefficients

- first term by van Ritbergen, Stuart

- now calculated through $\rho^{7}$ Pak, Czarnecki

- some logs were known Bauer, Falk, Luke

- $1 / \epsilon^{3}$ poles at $\rho^{4}$ and further $\rightsquigarrow$ additional terms of hardest masters integrals needed $+\ldots$ 


\section{Structure of the result}

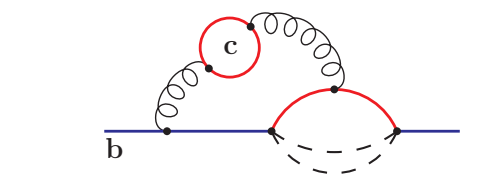$$
X_{c}=-\frac{1009}{280}+\frac{8}{3} \zeta_{3}+\frac{77}{216} \pi^{2}
$$$$
f \rho\left\{\frac{5}{4} \pi^{2}\right\}
$$$$
+\rho^{2}\left\{\frac{10}{3}+\frac{16}{3} \pi^{2}+\frac{52}{3} \ln \rho-8 \ln ^{2} \rho\right\}
$$$$
+\rho^{3}\left\{\frac{569}{36} \pi^{2}+\frac{64}{3} \pi^{2} \ln \rho\right\}
$$$$
+\rho^{4}\left\{-\frac{4483}{36}+196 \zeta_{3}+\frac{\pi^{2}}{6}+\left[\frac{73}{3} \pi^{2}+\frac{599}{6}\right] \ln \rho+44 \ln ^{2} \rho-32 \ln ^{3} \rho\right\}
$$$$
+\ldots
$$
with exact coefficients

- first term by van Ritbergen, Stuart Pak, Czarnecki

- some logs were known Bauer, Falk, Luke

- linear term unexpected

- expansion in $\rho=m_{c} / M_{b}$

- now calculated through $\rho^{7}$

- $1 / \epsilon^{3}$ poles at $\rho^{4}$ and further $\rightsquigarrow$ additional terms of hardest masters integrals needed 


\section{Mass definition}

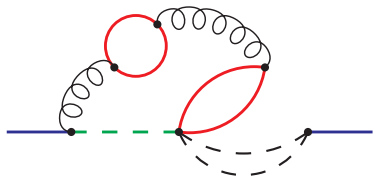

Origin of linear term:

region with soft momentum $\left(k \sim m_{q}\right)$

Calculation: pole mass scheme

QCD: soft region $m_{q} \sim \Lambda_{\mathrm{QCD}}$ cannot be treated perturbatively

Short-distance mass absorbs linear term:

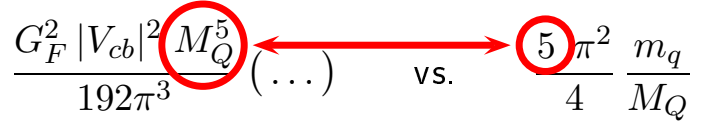

QED: perturbative at any scale $\rightsquigarrow$ pole mass for electron and muon Correction of $-0.43 \mathrm{ppm} \rightsquigarrow$ relevant for MuLan experiment 


\section{Summary}
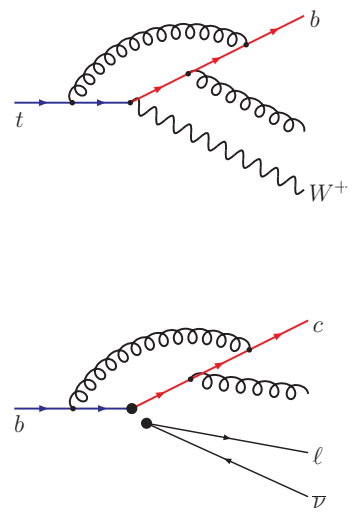

- applied optical theorem to polarised decay

- calculated helicity fractions of W at NNLO

- results relevant for LHC

- calculated NNLO mass effects for semi-leptonic decay rate and distribution moments

- results relevant for determination of $V_{c b}$ and $G_{F}$ 


\section{BLM}

estimate of higher order effects:

[Brodsky, Lepage, Mackenzie '82]

$$
\begin{aligned}
& \Gamma_{L}^{(3)}=-0.0186_{\mathrm{BLM}}+0.0043=-0.0143 \\
& \Gamma_{-}^{(3)}=-0.0052_{\mathrm{BLM}}+0.0013=-0.0039 \\
& \Gamma_{+}^{(3)}=0.00029_{\mathrm{BLM}}-0.00013=0.00016
\end{aligned}
$$




\section{Master integrals}

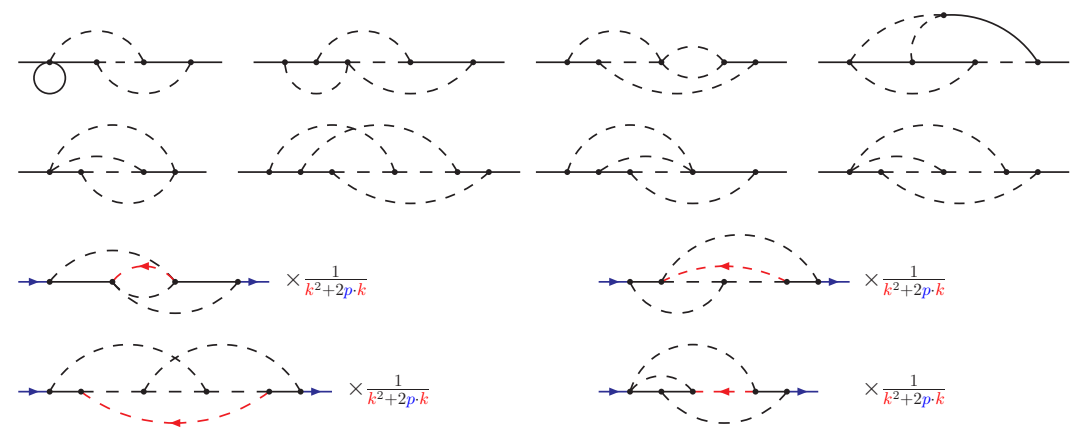

analytical results via:

- cutting rules

- differential equations

[Cutkosky '60]

[Kotikov '91] [Gehrmann, Remiddi '00] 


\section{Optical theorem}

Optical theorem relates decay width to imaginary part of top quark self-energy:
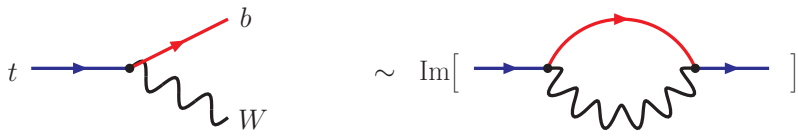

Advantages:

- real radiation is automatically taken into account

- "technology" for multi-loop calculations can be used 


\section{Expansion by regions}

general rules:

- use dimensional regularisation

- identify all regions which contribute to the given integral

- expand integrand in small quantities of each region

- set scaleless integrals to zero

- integrate loop momenta from $-\infty$ to $+\infty$

- sum contributions from all regions

[Smirnov, Applied Asymptotic Expansions in Momenta and Masses] 


\section{Higher orders}

consider both regions for all loop momenta

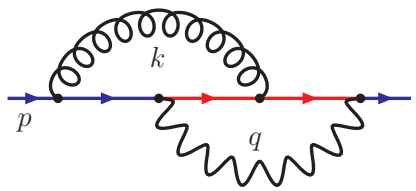

$\rightsquigarrow$ integrals become scaleless if gluon momentum is soft:

$$
\begin{aligned}
& \int \frac{\mathrm{d}^{d} k \mathrm{~d}^{d} q}{k^{2}\left(k^{2}+2 p \cdot k\right)(p+k+q)^{2}(p+q)^{2}\left(q^{2}-m_{W}^{2}\right)} \\
& \quad \sim \int \frac{\mathrm{d}^{d} k}{k^{2}(2 p \cdot k)} \times\left\{\begin{array}{ll}
\int \frac{\mathrm{d}^{d} q}{\left((p+q)^{2}\right)^{2} q^{2}} & q \text { hard } \\
\frac{1}{\left(p^{2}\right)^{2}} \int \frac{\mathrm{d}^{d} q}{\left(q^{2}-m_{W}^{2}\right)} & q \text { soft }
\end{array}+\ldots\right.
\end{aligned}
$$




\section{Treatment of $\gamma_{5}$}

- replace $\gamma_{5}$ with $\varepsilon$ tensor:

$$
\gamma_{\mu} \gamma_{5} \rightarrow \frac{i}{3 !} \varepsilon_{\mu \alpha \beta \delta} \gamma^{\alpha} \gamma^{\beta} \gamma^{\delta}
$$

- contract $\varepsilon$ tensor with projector $\rightsquigarrow$ metric tensors

- renormalise axial-vector current

- restore anti-commutativity with additional finite renormalisation 


\section{Unpolarised case}

average over spin states of top quark

$$
\bar{u}(p)[\ldots] u(p) \rightarrow \frac{1}{2} \operatorname{Tr}\left\{\left(\not p+m_{t}\right)[\ldots]\right\}
$$

sum over polarisations of $\mathrm{W}$ boson

$$
\text { polarisations }
$$

$$
\epsilon_{\mu}^{*}(q) \epsilon_{\nu}(q)=-\left(g_{\mu \nu}-\frac{q_{\mu} q_{\nu}}{m_{W}^{2}}\right)
$$

$\rightarrow$ numerator of $\mathrm{W}$ propagator in unitary gauge 


\section{Longitudinally polarised W boson}

\section{kinematics}

top quark is at rest:

$$
p^{\mu}=\left(m_{t}, 0,0,0\right)
$$

W momentum in $z$ direction: $q^{\mu}=\left(q_{0}, 0,0,|\vec{q}|\right)$

polarisation vector:

$$
\epsilon_{L}^{\mu}(q)=\left(|\vec{q}|, 0,0, q_{0}\right) / m_{W}
$$

$$
\begin{gathered}
\Rightarrow \epsilon_{L}^{\mu}=-\frac{m_{W}^{2} p^{\mu}-p \cdot q q^{\mu}}{m_{W} m_{t}|\vec{q}|} \\
\rightsquigarrow \epsilon_{L}^{\mu *}(q) \epsilon_{L}^{\nu}(q)=\frac{\left(m_{W}^{2} p^{\mu}-p \cdot q q^{\mu}\right)\left(m_{W}^{2} p^{\nu}-p \cdot q q^{\nu}\right)}{m_{W}^{2} m_{t}^{2}|\vec{q}|^{2}}
\end{gathered}
$$




\section{Polarisation}

use projection operators:

$$
\begin{aligned}
\mathbb{P}_{0}^{\mu \nu}(q) & =-g^{\mu \nu}+\frac{q^{\mu} q^{\nu}}{m_{W}^{2}} \\
\mathbb{P}_{L}^{\mu \nu}(q) & =\frac{\left(m_{W}^{2} p^{\mu}-p \cdot q q^{\mu}\right)\left(m_{W}^{2} p^{\nu}-p \cdot q q^{\nu}\right)}{m_{W}^{2} m_{t}^{2}|\vec{q}|^{2}} \\
\mathbb{P}_{F}^{\mu \nu}(q) & =-\frac{i}{m_{t}|\vec{q}|} \varepsilon^{\mu \nu \sigma \rho} p_{\sigma} q_{\rho} \\
\mathbb{P}_{ \pm}^{\mu \nu} & =\mathbb{P}_{0}^{\mu \nu}-\mathbb{P}_{L}^{\mu \nu} \pm \mathbb{P}_{F}^{\mu \nu}
\end{aligned}
$$

complications:

- appearance of $|\vec{q}|=\sqrt{q_{0}^{2}-m_{W}^{2}}$ in denominator

- treatment of $\gamma_{5}$ in dimensional regularisation 